\title{
Single Pulse Frequency Compounding Protocol for Superharmonic Imaging.
}

\author{
Mikhail G. Danilouchkine ${ }^{a, b}$, Paul L. M. J. van Neer ${ }^{a}$, Guillaume M. Matte ${ }^{a}$, Martin \\ D. Verweij ${ }^{c}$, Nico de Jong ${ }^{a, b}$ \\ ${ }^{a}$ Department of Biomedical Engineering, Erasmus Medical Center, Ee2302, \\ P.O.Box 2040, 3000 CA, Rotterdam, the Netherlands. \\ ${ }^{b}$ Interuniversity Cardiology Institute of the Netherlands, \\ P.O.Box 19258, 3501 DG, Utrecht, the Netherlands. \\ ${ }^{c}$ Delft University of Technology, \\ 2628 CD, Delft, the Netherlands.
}

\begin{abstract}
Second harmonic imaging is currently adopted as standard in commercial echographic systems. A new imaging technique, coined as superharmonic imaging (SHI), combines the 3rd till the 5th harmonics, arising during nonlinear sound propagation. It could further enhance resolution and quality of echographic images. To meet the bandwidth requirement for SHI a dedicated phased array has been developed: a low frequency subarray, intended for transmission, interleaved with a high frequency subarray, used in reception. As the bandwidth of the elements is limited, the spectral gaps in between the harmonics cause multiple reflection artifacts. Recently, we introduce a dual-pulse frequency compounding (DPFC) method to suppress those artifacts at price of a reduced frame rate. In this study we investigate the feasibility of performing the frequency compounding protocol within a single transmission.

The traditional DPFC method constructs each trace in a post-processing stage by summing echoes from two emitted pulses, the second slightly frequency-shifted compared to the first. In the newly proposed method, the transmit aperture is divided into two parts: the first half is used to send a pulse at the lower center frequency, while the other half simultaneously transmits at the higher center frequency. The suitability of the protocol for medical imaging applications in terms of the steering capabilities was performed in a simulation study using the FIELD II toolkit. Moreover, an experimental study was performed to deduce the optimal parametric set for implementation of the clinical imaging protocol. The latter was subsequently used to obtain the images of a tissue mimicking phantom containing strongly reflecting wires. For in-vitro acquisitions the SHI probe with interleaved phased array (44 odd elements at $1 \mathrm{MHz}$ and 44 even elements at $3.7 \mathrm{MHz}$ elements, optimized for echocardiography) was connected to a fully programmable ultrasound system.
\end{abstract}

The results of the Field II simulations demonstrated that the angle between the main and grating lobe amounted to $90^{\circ}$. The difference in the fundamental pressure level between those lobes was equal to $-26.8 \mathrm{~dB}$. Those results suggest that the superharmonic content in the grating lobe was acceptably low. A considerable improvement in the axial resolution of the SHI component $(0.73 \mathrm{~mm})$ at $-6 \mathrm{~dB}$ in comparison with the 3rd harmonic $(2.23 \mathrm{~mm})$ was observed. A similar comparison in terms of the lateral resolution slightly favored the superharmonic component by $0.2 \mathrm{~mm}$. Additionally, the images of the tissue mimicking phantom exhibited an absence of the multiple reflection artifacts in the focal and post-focal regions.

The new method is equally effective in eliminating the ripple artifacts associated with SHI as the dual pulse technique, while the full frame rate is maintained.

This work was funded by the Dutch foundation for technical sciences (STW) and OlDelft. (Delft, the Netherlands).

Medical Imaging 2011: Ultrasonic Imaging, Tomography, and Therapy, edited by Jan D'hooge, Marvin M. Doyley, Proc. of SPIE Vol. 7968,796808 - (C) 2011 SPIE · CCC code: 1605-7422/11/\$18 · doi: 10.1117/12.877699 


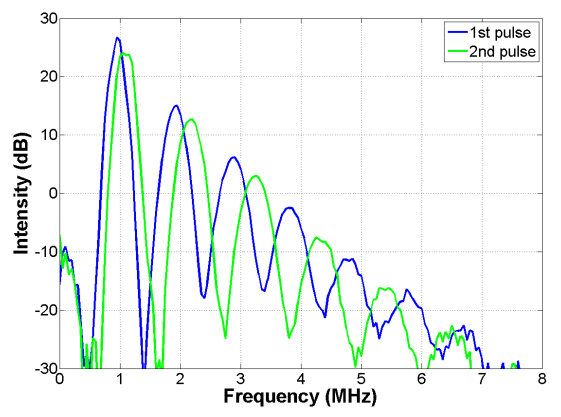

Figure 1. The concept of the frequency compounding method for SHI.

\section{INTRODUCTION}

Utilization of the nonlinear properties of the medium to generate harmonics dates back to the work of Kompfner et al. ${ }^{1}$ on acoustic microscopy. The relevance of this phenomenon in the context of medical applications was later demonstrated by Muir ${ }^{2}$ and experimentally confirmed by Starritt. ${ }^{3}$ These two discoveries spawned the new era of harmonic imaging in medical ultrasound. To date, this technique has become the de-facto standard for studying the nonlinear behavior of ultrasound contrast agents (UCAs). From a medical point of view it allows for the detection of the perfused or non-perfused tissue region for the purposes of the functional assessment of anatomic organs. Until recently clinical protocols were limited to the second harmonic only.

A few years ago a new method for tissue and UCA imaging was proposed by Bouakaz et al. ${ }^{4}$ It was based on the selective imaging of multiple harmonics (from the 3rd up to the 5th). The method was coined as superharmonic imaging (SHI). The new technique supposedly inherits the merits of second harmonic imaging, but further improves the spatial resolution and reduces the level of the side lobes. Indeed, the first experimental comparison of the B-mode scans of the bladder cavity, obtained with second and superharmonic imaging, have proven the superiority of the latter method. ${ }^{5}$ The inner fluid-filled cavity appeared not only more hypoenhanced, but also exhibited a less visible granular pattern. Moreover, the simulation results initially showed that the energy content of the second and super harmonic components are similar in the far-field..$^{5,6}$

In spite of the apparent advantages of SHI, there exists a fundamental problem associated with this technique. It is related to the fact that the amplitude of the higher harmonics is progressively lower than one of the fundamental. ${ }^{3}$ Each individual component of the higher harmonics has an inherently low signal-to-noise ratio. Hence, their contribution should be combined over the entire superharmonic band to achieve an energy level sufficient for imaging. The spectrum of a nonlinearly propagated signal corresponding to the initial 3-cycle apodized sinus burst, commonly applied in medical imaging protocols, is shown in fig. 1. The global maximum corresponds to the fundamental frequency of the transmitted pulse. The local maxima at multiples of the fundamental frequency are the high order harmonics. The existence of the local minima in between the harmonics causes ripples in the point spread function (PSF). This gives rise to ghost reflections along the direction of the wave propagation and makes imaging impossible in a practical situation.

\section{BACKGROUND}

To advance SHI into the clinical environment, a dual pulse frequency compounding (DPFC) technique was proposed by Matte et al. ${ }^{7}$ The main idea is also summarized in fig. 1. The imaging protocol makes use of two transmitted pulses with the second pulse of slightly different frequency. Consequently, the temporal traces are summed together in the post-processing stage. The choice of the frequency shift is of the utmost importance for the frequency compounding protocols. Namely, the location of the higher harmonics in the second pulse should fill the spectral gaps of the first pulse as shown in fig. 1. The spectrum of the cumulative temporal trace exhibits less prominent minima and has a relatively smooth appearance of the superharmonic band. In terms of the imaging characteristics that would yield a better PSF. The frequency shift was theoretically predicted with the Fubini solution and experimentally validated with the simulation and measurement on a single element transducer. ${ }^{7}$ Although a good agreement between the simulated and theoretically predicted frequency shift was 


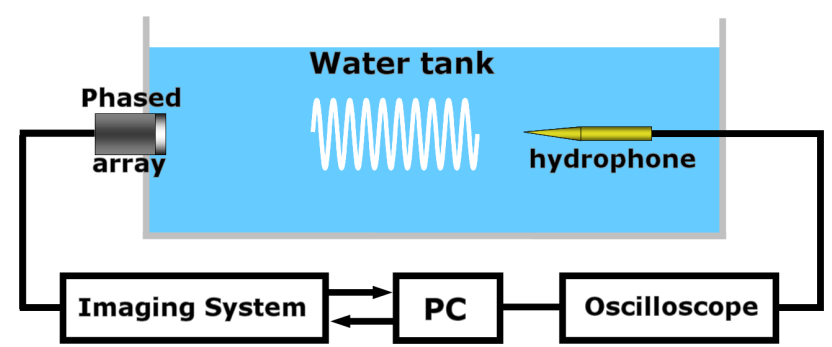

Figure 2. The experimental setup used in combination with the dual-frequency phased array transducer.

observed, the measurement demonstrated that the suppression of the ripple artifacts with the single element transducer had not reached its optimal level.

In the most recent contribution ${ }^{8}$ the DPFC SHI technique was adapted for a phased-array transducer prototype. ${ }^{9,10}$ The experimental measurements demonstrated a marked improvement in the axial resolution and a slight increase in the lateral resolution of the superharmonic PSF in comparison with one of the 3rd harmonic. Additionally, the authors have observed an adequate level of suppression of the ghost reflection artifacts. Nevertheless, the investigated protocol suffers from a reduced frame rate as it had been implemented as a dual transmission. The multiple pulse techniques are generally known to be susceptible to motion artifacts and, thus, problematic in contrast agent studies.

The research focus of this paper is to investigate a possibility of implementing the frequency compounding method in a single transmission. It is coined as a single pulse frequency compounding (SPFC) protocol. In order to achieve this goal the main objectives should be met:

- the steering capabilities of such an imaging protocol should be established in order to guarantee its usability in the clinical practice;

- to achieve artifact-free imaging an optimal frequency and spectral shift have to be found.

In this paper feasibility of the SPFC protocol on a medical phased-array transducer will be demonstrated and validated in-vitro.

\section{MATERIALS AND METHODS}

\subsection{SPFC implementation}

In medical ultrasound short oscillating apodized bursts are commonly used for imaging. The duration of an imaging pulse is typically between 3 and 4 cycles. In the post-processing phase envelope detection is applied to construct an ultrasound image. In our previous study, ${ }^{8}$ the influence of the pulse duration on the performance of the frequency compounding was investigated. A 4-cycle burst appeared to be infeasible, as it is a relatively narrow banded pulse. The range of the suitable pulse durations turned out to lie between 2 and 3 cycles. The SPFC protocol has to cope with the lower level of the energy intensity compared to its dual-pulse counterpart. Therefore, 2 and 2.5-cycles are disregarded from this study and the 3-cycle burst will only be considered.

The idea behind the SPFC implementation is to split the transmit aperture into two subapertures. One part emits a pulse at a lower center frequency, while the other half transmits a burst at a higher center frequency. A $130 \%$ bandwidth is necessary to guarantee transmission and reception over five harmonic bands, which is considerably larger than that achievable with conventional arrays. Hence, an approach based on the separated acoustic stacks was adhered to. The interleaved design of the phased array featured the alternating transmit and receive elements and yielded the best beam characteristics. ${ }^{11}$ The implementation of the SPFC protocol implies a further split of the transmit aperture and results in a sparser arrangement of the elements emitting the same frequency component. Hence, the feasibility of the SPFC implementation in terms of its steering capabilities should be carefully investigated. 


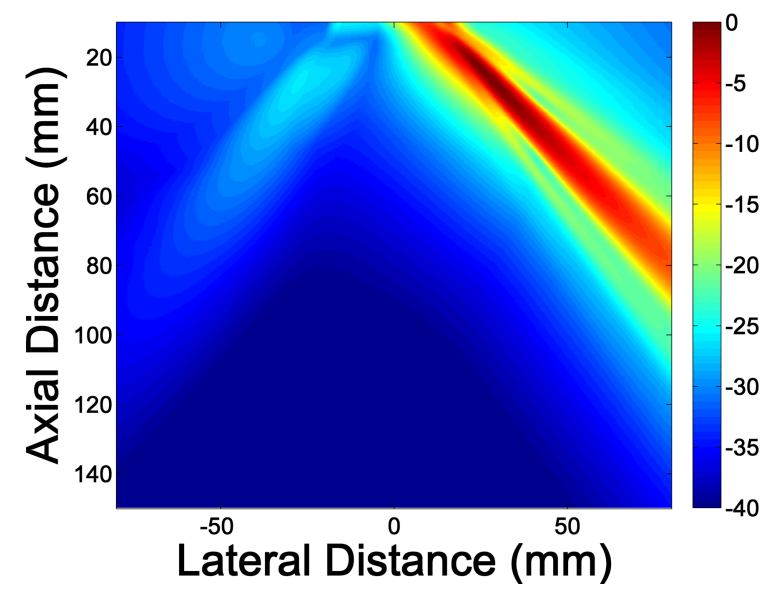

Figure 3. The spatial distribution of the fundamental peak pressure for a steered beam, generated with the Field II simulation. The pressure levels are normalized with respect to the maximal intensity and expressed in the logarithmic scale with the color coded scheme.

\subsection{Steering capabilities of SPFC}

A study of the steered beams with the SPFC protocol was conducted numerically. The common computational approach comprises a so-called KZK model ${ }^{12}$ to predict the nonlinear propagation of ultrasound waves in a medium. However, this approach is only valid for the paraxial approximation in a narrow spherical cone around the acoustical axis of the transducer. Instead of using a nonlinear model, the feasibility of the SPFC protocol in terms of steering capabilities was based on the spatial distribution of the fundamental peak pressure and utilized the Field II toolkit. ${ }^{13,14}$

The simulations were performed for a phased-array transducer with the element size $13 \mathrm{~mm} \times 0.2 \mathrm{~mm}$ and pitch $0.5 \mathrm{~mm}$. The lateral and elevation foci were set at a distance of $6 \mathrm{~cm}$ from the transducer surface. It transmitted Gaussian apodized 3-cycle sine bursts with center frequencies of $0.86 \mathrm{MHz}$ and $1.16 \mathrm{MHz}$. The propagation medium was water. The parameters used were: an ambient density $\left(\rho_{0}\right)$ of $1000 \mathrm{~kg} / \mathrm{m}^{3}$, a small-signal acoustic wave speed $c_{0}$ of $1490 \mathrm{~m} / \mathrm{s}$, a nonlinearity parameter $\beta$ of 3.5 and an attenuation $(\alpha)$ of $0.22 \mathrm{~dB} \cdot \mathrm{m}^{-1} \cdot \mathrm{MHz}^{-2} \cdot{ }^{15}$ The beam was steered at $45^{\circ}$.

\subsection{Hydrophone setup}

The experimental setup, depicted in fig. 2, featured a water-filled tank, a hydrophone (diameter $0.2 \mathrm{~mm}$, Precision Acoustics, Dorchester, UK) mounted in a XYZ-system. The signals received by the hydrophone were digitized by an oscilloscope (9400A, Lecroy, Geneva, Switzerland) with a sampling frequency of $100 \mathrm{MHz}$ and transferred to a computer for further processing. The dual-frequency phased array, optimized for SHI, ${ }^{10}$ was mounted on the tank's sidewall. The odd elements in the transmit aperture were excited by Gaussian apodized 3-cycle sine bursts of varying lower center frequencies. The sweep was performed between $0.78 \mathrm{MHz}$ and $1.0 \mathrm{MHz}$. The even elements emitted the same pulse at a higher center frequency, ranging $1.0 \mathrm{MHz}$ and $1.3 \mathrm{MHz}$.

\subsection{Tissue mimicking phantom setup}

The dual-frequency phased array transducer in combination with the ultrasound system was used to make Bmode images of a tissue mimicking phantom (model 040GSE, CIRS Inc., Norfolk, USA). The section of the phantom with an attenuation of $0.5 \mathrm{~dB} \cdot \mathrm{cm}^{-1} \cdot \mathrm{MHz}^{-1}$ was scanned. The excitation signals were produced by a multi-channel programmable ultrasound system (OPEN system, Lecoeur Electronique, Chuelles, France, first reported $\left.i^{16}\right)$. The transducer was excited by 3 -cycle Gaussian apodized sine bursts with center frequencies $0.86 \mathrm{MHz}$ and $1.16 \mathrm{MHz}$ and amplitude of $108 \mathrm{Vpp}$. 


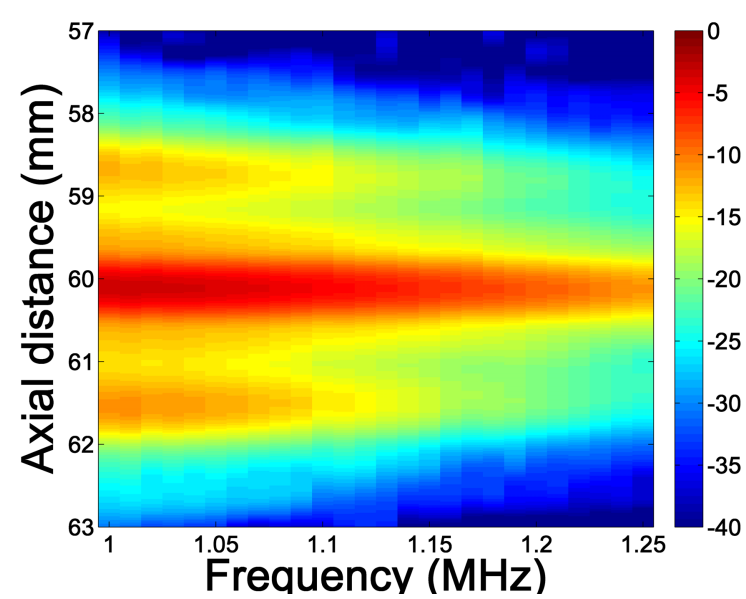

(a) The axial intensity profiles

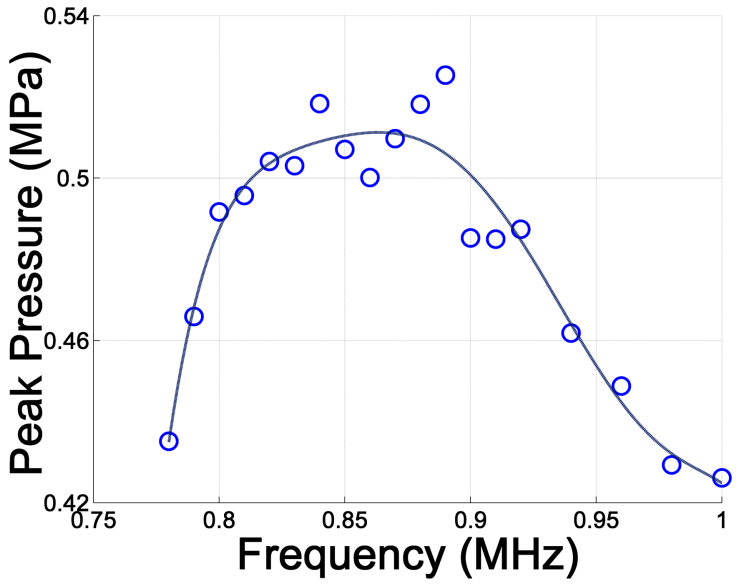

(b) The peak pressure profile

Figure 4. SPFC optimization plots. (a).The axial intensity profiles (vertical axis) versus the higher center frequency (horizontal axis) for a 3-cycle transmission. The lower center frequency was fixed at $0.86 \mathrm{MHz}$, while the higher center frequency varied between 1.0 and $1.25 \mathrm{MHz}$.(b). The relationship between the peak pressure (the vertical axis) of the superharmonic component and the low center frequency for optimized protocols. The dots represent the measured peak pressure values, while the solid line corresponds to the polynomial fit of the 6 th order through the measurements.

\section{EXPERIMENTS AND RESULTS}

\subsection{Simulation of steered beam}

The spatial distribution of the fundamental pressure for the steered beam, computed with Field II, is shown in fig. 3. The peak pressure values were normalized to the maximal amplitude at focus and expressed in the logarithmic scale. The main lobe corresponds to a location at $0 \mathrm{~dB}$, while the local maximum of the peak pressure, located symmetrically across the transducer's axis (the vertical line at $0 \mathrm{~mm}$ of the lateral dimension in the figure), indicates the position of the grating lobe. The angular distance between those lobes amounted to approximately $90^{\circ}$. The peak pressure at the grating lobe was lower by $26.8 \mathrm{~dB}$.

\subsection{Optimal frequencies}

The pressure intensity along the axial dimension versus the higher center frequency is shown in fig. 4(a) for 3-cycle transmissions and the fixed lower center frequency at $0.86 \mathrm{MHz}$. The graduate vanishing of the ghost reflection artifacts was observed as the spectral shift between the emitted pulse increased. The peak intensity coincides with the location of the lateral focus at the depth of $6 \mathrm{~cm}$. The ripple artifacts manifest as the local maxima in the pre- and post-focal regions for the range of higher center frequency between $1.00 \mathrm{MHz}$ and $1.15 \mathrm{MHz}$. As the spectral gap increases those artifacts become less prominent and disappear at $1.16 \mathrm{MHz}$, corresponding to the optimal spectral shift.

The range of the lower center frequencies, for which the optimal performance of the SPFC protocol is achieved, can be deduced from the plot in fig. 4(b). For each lower center frequency in the range between $0.78 \mathrm{MHz}$ and $1.0 \mathrm{MHz}$ the optimal spectral shift was determined using the method described in the previous paragraph. Given the optimal spectral shift, the absolute value of the peak pressure at focus was measured. Fig. 4(b) summarizes the dependency between the peak pressure and the lower center frequency. The best performance of the DPFC protocol was observed in the range of lower center frequencies between 0.83 and $0.88 \mathrm{MHz}$, where the peak pressure reached maximal of $0.5 \mathrm{MPa}$ for the SHI component. The optimal spectral shift amounted to $300 \mathrm{kHz}$.

\subsection{PSF comparison}

The visual appearance of the third harmonic PSF and the SHI PSF's with and without the frequency compounding is shown in fig. 5. The PSF's are depicted as the spatial intensity profile versus the axial and lateral dimensions for 3 cycle transmissions. The intensities were normalized to their respective maxima. The PSFs were 


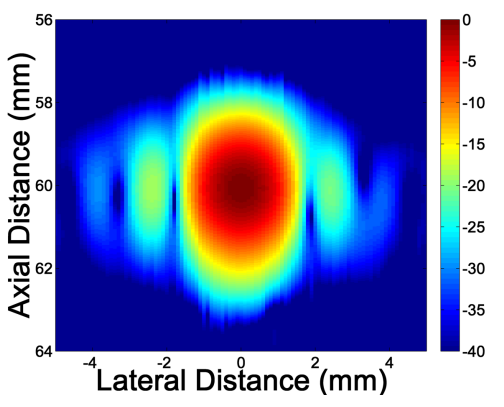

(a) 3rd Harmonic

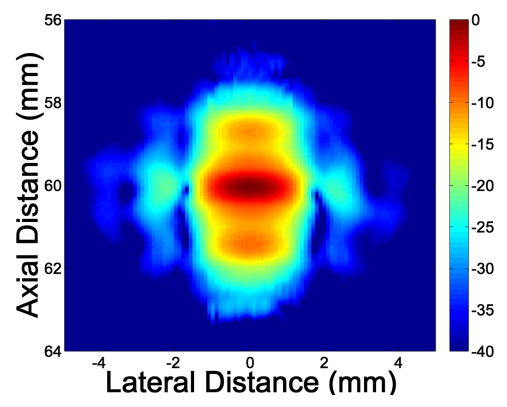

(b) SHI without compounding

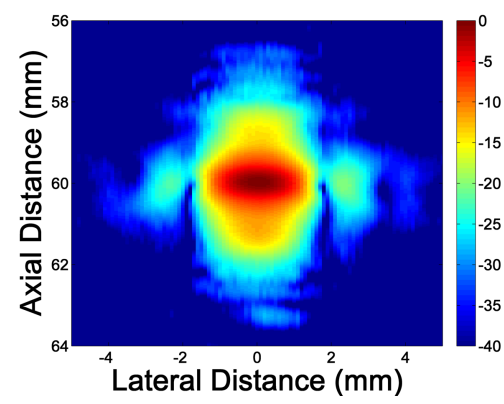

(c) SHI with compounding

Figure 5. Comparison of PSF's for 3rd harmonic (left panel), superharmonic without the frequency compounding (middle panel), and superharmonic for the optimal frequency compounding (right panel). The relative intensity is color coded in the imaging range between 0 and $-40 \mathrm{~dB}$.

strikingly similar in terms of the intensity roll-off along the lateral dimension. The differences are clearly visible along the direction of the wave propagation. The largest extent of the PSF in the axial direction is observed for the 3rd harmonic PSF. The PSF for SHI with and without the frequency compounding are similar around the focus. However, the SHI PSF without the frequency compounding exhibits the ripple artifacts, appearing as a local maxima of $-10 \mathrm{~dB}$ and $-12 \mathrm{~dB}$. The ripple in the pre-focal region disappears for the SHI PSF with the frequency compounding. The post-focal artifact, however, turned into a plateau at $-14 \mathrm{~dB}$. The SHI with the frequency compounding, lateral beam widths at $-6 \mathrm{~dB}$ and $-20 \mathrm{~dB}$ were $1.9 \mathrm{~mm}$ and $3.1 \mathrm{~mm}$, respectively. For the third harmonic, these parameters measure $2.1 \mathrm{~mm}$ and $3.2 \mathrm{~mm}$, respectively. The axial beam widths at $-6 \mathrm{~dB}$ and $-20 \mathrm{~dB}$ were $0.73 \mathrm{~mm}$ and $3.8 \mathrm{~mm}$, respectively. The same parameters amounted to $2.23 \mathrm{~mm}$ and $4.45 \mathrm{~mm}$ for the third harmonic.

\subsection{In-vitro imaging}

Fig. 6 shows the B-mode SHI images of a tissue mimicking phantom created with (right) and without (left) the frequency compounding. For the latter image, all transmit elements emitted a 3-cycle burst at $1.0 \mathrm{MHz}$ center frequency. The former image generated with the SPFC protocol using the lower center frequency of $0.86 \mathrm{MHz}$, the spectral shift of $300 \mathrm{kHz}$ and the 3-cycle transmission. The ghost reflections are clearly visible on the SHI image without the frequency compounding. The image is distorted as the ripple artifacts even destroy the speckle patter outside the bright spots, corresponding to wire reflectors inside the phantom. This pattern is restored with the frequency compounding. The wires in the focal and post-focal areas also appeared to be free from the ghost reflection artifacts, while the wire in the pre-focal region is still corrupted. This effect is probably caused by the insufficient power of imaging harmonics, as they are building up during the wave propagation and reach a higher level level deeper in the axial direction.

\section{DISCUSSION}

The optimal performance of the SPFC protocol was observed for the spectral ranges between $0.83 \mathrm{MHz}$ and $0.88 \mathrm{MHz}$ and between $1.13 \mathrm{MHz}$ and $1.18 \mathrm{MHz}$ for the lower and higher center frequencies, respectively. The average center frequency lies between $0.98 \mathrm{MHz}$ and $1.03 \mathrm{MHz}$. The transmit transfer function for the phasedarray transducer was experimentally measured and presented in our previous contribution. ${ }^{10}$ The maximum of the center frequency occurs around $1.0 \mathrm{MHz}$ with the graduate decrease in transmit efficiency towards the lower and higher frequencies. Hence, the optimal center frequencies for the SPFC protocol are situated symmetrically around the peak of the transducer's transfer efficacy. On the other hand, the harmonic level is determined by two competing phenomena: nonlinear propagation and frequency dependent attenuation. ${ }^{17}$ Thus, the optimal transfer frequency for SHI imaging is also tight to an applications domain and can lie above $1.0 \mathrm{MHz} .{ }^{11,17}$ The SPFC protocol would need adaptations for a different frequency range. The experimental optimization can follow the guidelines described in this contribution. 


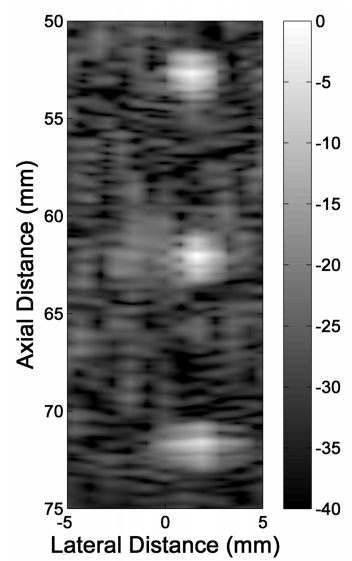

(a) SHI without compounding

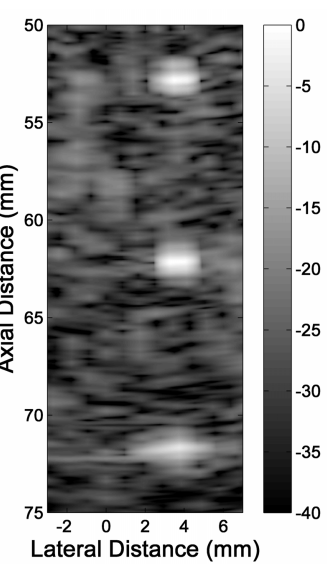

(b) SHI with compounding

Figure 6. The B-mode images of a tissue mimicking phantom created with SHI scheme without (left) and with (right) the frequency compounding. The transducer was located at the top of the image. The relative intensity is encoded by a gray scale using a dynamic range of $40 \mathrm{~dB}$.

The results, reported for the SPFC protocol, reasonably agree with the ones for the dual-pulse technique. The lateral and axial resolutions at $-6 \mathrm{~dB}$ exhibit a perfect match, while the lateral resolution at $-20 \mathrm{~dB}$ amounts to $3.1 \mathrm{~mm}$ and $3.4 \mathrm{~mm}$ for the SPFC and DPFC protocol, respectively. The axial resolution at the same level for the DPFC method appears to be better. In terms of the optimal parameters, the lower center frequency differs by $400 \mathrm{kHz}$ between the protocols, while the spectral shift differs insignificantly. Those discrepancies can be attributed to the subjectivity of a human observer during the assessment procedure.

In this study, we resorted to a simplified approach for the estimation of the steering capabilities of the SPFC protocol, based on the Field II simulations of the fundamental pressure distribution. The peak pressure in the grating lobe turned out to be considerable lower than one in the main lobe. Thus, the initial pressure, emitted in the direction of this direction, is considerably less, yielding a lower level of harmonics. From this we postulated that the protocol is suitable for medical application in terms of the dynamic range. Nevertheless, more sophisticated numerical methods can be employed to accurate predict the ratio of harmonics in the main and grating lobes and assessing the lower bound of the possible dynamic range. For example, the Iterative Nonlinear Contrast Source ${ }^{18,19}$ technique is highly suitable for these purposes and have been proven to provide accurate numerical simulations for steered nonlinearly propagating ultrasound beams.

\section{CONCLUSION}

The new SPFC method is equally effective in eliminating the ripple artifacts associated with SHI as the previously presented dual pulse technique, but maintains the full frame rate. Our findings confirm that the transfer functions of both transducer and system have to be taken into account to determine the optimal transmission frequencies for the frequency compounding SHI protocols. The optimal results with the frequency compounding were achieved for a 3-cycle pulse and transmission frequencies of $0.86 \mathrm{MHz}$ and $1.16 \mathrm{MHz}$. Moreover, it has markedly improved axial and lateral resolutions compared to the third harmonic.

\section{ACKNOWLEDGEMENT}

The authors would like to thank OlDelft (Delft, The Netherlands) for providing the financial and technical support for the project. The financial contribution of Dutch foundation for technical sciences (STW) is also greatly appreciated. 


\section{REFERENCES}

[1] Kompfner, R. and Lemons, R. A., "Nonlinear acoustic microscopy," Applied Physics Letters 28(6), 295-297 (1976).

[2] Muir, T. G. and Carstensen, E. L., "Prediction of nonlinear acoustic effects at biomedical frequencies and intensities," Ultrasound in Medicine and Biology 6(4), 345-357 (1980).

[3] Starritt, H. C., Duck, F. A., Hawkins, A. J., and Humphrey, V. F., "The development of harmonic distortion in pulsed finite-amplitude ultrasound passing through liver," Physics in Medicine and Biology 31(12), 14011409 (1986).

[4] Bouakaz, A., Frigstad, S., Ten Cate, F. J., and de Jong, N., "Super harmonic imaging: A new imaging technique for improved contrast detection," Ultrasound in Medicine and Biology 28(1), 59-68 (2002).

[5] Bouakaz, A. and de Jong, N., "Native tissue imaging at superharmonic frequencies," IEEE Transactions on Ultrasonics Ferroelectrics and Frequency Control 50(5), 496-506 (2003).

[6] Ma, Q. Y., Zhang, D., Gong, X. F., and Ma, Y., "Investigation of superharmonic sound propagation and imaging in biological tissues in vitro," Journal of the Acoustical Society of America 119(4), 2518-2523 (2006).

[7] Matte, G., van Neer, P., Borsboom, J., Verweij, M., and de Jong, N., "A new frequency compounding technique for super harmonic imaging," in [Ultrasonics Symposium], Proc. IEEE, 357-360 (2008).

[8] van Neer, P., Danilouchkine, M., Matte, G., Verweij, M., and de Jong, N., "Dual pulse frequency compounded super harmonic imaging for phased array transducers," in [Ultrasonics Symposium], Proc. IEEE, 381-384 (2009).

[9] Bouakaz, A., ten Cate, F. J., and de Jong, N., "A new ultrasonic transducer for improved contrast nonlinear imaging," Phys. Med. Biol. 49(16), 35153525 (2004).

[10] van Neer, P., Matte, G., Danilouchkine, M., Prins, C., van den Adel, F., and de Jong, N., "Super harmonic imaging: development of an interleaved phased array transducer," IEEE Trans. Ultrason. Ferroelectr. Freq. Control 57(2), 455-468 (2010).

[11] Matte, G., van Neer, P., Danilouchkine, M., Huijssen, J., Verweij, M., and de Jong, N., "Optimization of a phased-array transducer for multiple harmonic imaging in medical applications: Frequency and topology," IEEE Trans. Ultrason. Ferroelectr. Freq. Control 58(3) (2011).

[12] Zabolotskaya, E. and Khokhlov, R., "Quasi-plane waves in the nonlinear acoustics of confined beams," Soviet Physics - Acoustics 15, 3540 (1969).

[13] Jensen, J. and Svendsen, N., "Calculation of pressure field from arbitrarily shaped, apodized and excited ultrasound transducers," IEEE Trans. Ultrason. Ferroelectr. Freq. Control 39(2), 262-267 (1992).

[14] Jensen, J., "Field: A program for simulating ultrasound systems," Paper presented at the 10th Nordic-Baltic Conference on Biomedical Imaging Published in Medical \&3 Biological Engineering \& Computing 34(Supp. 1 Pt. 1), 351-353 (1996).

[15] Duck, F., [Physical Properties of Tissues], Academic Press, Inc (1990).

[16] Vignon, F., Aubry, J.-F., Tanter, M., Margoum, A., M.Fink, and Lecoeur, J., "Dual-arrays brain imaging prototype: Experimental in vitro results," in [Ultrasonics Symposium], Proc. IEEE, 504-507 (2005).

[17] van Neer, P., Danilouchkine, M., Matte, G., Voormolen, M., Verweij, M., and de Jong, N., "A comparative study of optimal fundamental, second- and superharmonic imaging," in [Ultrasonics Symposium], Proc. IEEE (2010).

[18] Huijssen, J., Verweij, M., and de Jong, N., "3d time-domain modeling of nonlinear medical ultrasound with an iterative greens function method," in [Ultrasonics Symposium], Proc. IEEE, 2124-2127 (2006).

[19] Huijssen, J., Verweij, M., and de Jong, N., "Greens function method for modeling nonlinear threedimensional pulsed acoustic fields in diagnostic ultrasound including tissue-like attenuation," in [Ultrasonics Symposium], Proc. IEEE, 375-378 (2008). 